\title{
Absent posterior interventricular artery
}

\author{
J.N. Bridgeman, P.J. Adds \\ Division of Biomedical Sciences (Anatomy), St. George's University of London, United Kingdom
}

[Received 11 June 2014; Accepted 7 July 2014]

During the dissection of the thorax of a 79-year-old Caucasian male cadaver, the posterior interventricular coronary artery was found to be completely absent. Congenital heart abnormalities are frequent but absent arteries are uncommon and when occurring are often associated with replacement vessels from the existing circulation, making complete absence rare. This condition may well have been asymptomatic in life, but such variations could have serious implications in patients with underlying heart pathology. (Folia Morphol 2015; 74, 3: 396-398)

Key words: heart, coronary circulation, posterior interventricular artery, variant

\section{INTRODUCTION}

The posterior interventricular artery is one of the main subdivisions of the cardiac vasculature. The origin of the posterior interventricular artery accounts for the termed dominance of the heart. The main function of the posterior interventricular artery is to supply a small posterior aspect of the interventricular septum [7] and the posterior aspect of the right ventricle [6].

Variations in the cardiac vasculature are common, affecting around $1.3 \%$ of the population undergoing angiography, with around $80 \%$ of these being asymptomatic [5]. These abnormalities include ectopic ostia of the coronary arteries [4] and myocardial bridges [1]. The right coronary artery has been described as the coronary vessel most affected by congenital abnormalities [9].

\section{CASE REPORT}

A dissection was performed on the thoracic viscera of a 79-year-old Caucasian male formalin-fixed cadaveric body. The cause of death was listed as metastatic splenic flexure tumour (a non-coronary cause). The patient had a history of atrial fibrillation and high blood pressure, for which he was taking amlodipine, digoxin and clopidogrel. The patient had never reported experiencing chest pain, had a normal exercise tolerance and was an ex-smoker.

The anterior thoracic wall was opened and the heart and lungs were removed together. The heart was dissected to reveal the 4 cardiac valves and the coronary vasculature. The right and left coronary arteries emerged from the right and left coronary sinus respectively and not ectopically. The right coronary artery then continued to become the right circumflex artery. The left main trunk gave rise to the left anterior descending artery and then the left marginal and the left circumflex arteries. The left anterior descending artery extended down to the apex where it continued to travel up a small section of the posterior interventricular sulcus (Fig. 1).

At this point several variations were noted. As the left circumflex artery continued around the atrio-ventricular groove it took a perpendicular path towards the apex of the heart on the left side as opposed to its normal path to the posterior aspect of the left ventricle. This artery also had a myocardial bridge about halfway down (Fig. 2).

Those were all the branches found from the left coronary artery. The right coronary artery was also slightly abnormal due to the fact that it terminated with the circumflex branch. On the posterior aspect

Address for correspondence: Mr P.J. Adds, Division of Biomedical Sciences (Anatomy), St. George's University of London, Cranmer Terrace, London SW17 ORE, United Kingdom, tel: +44(0)2087255208, e-mail: padds@sgul.ac.uk 


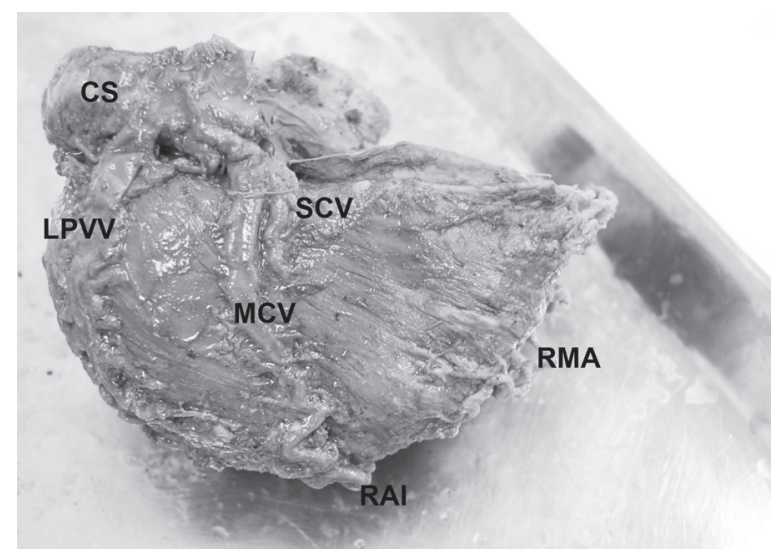

Figure 1. The posterior aspect of the heart with the atria removed; CS - coronary sinus; LPVV — left posterior ventricular vein; $\mathrm{MCV}$ - middle cardiac vein; RAI — recurrent anterior interventricular artery; RMA — right marginal artery; SCV — small cardiac vein.

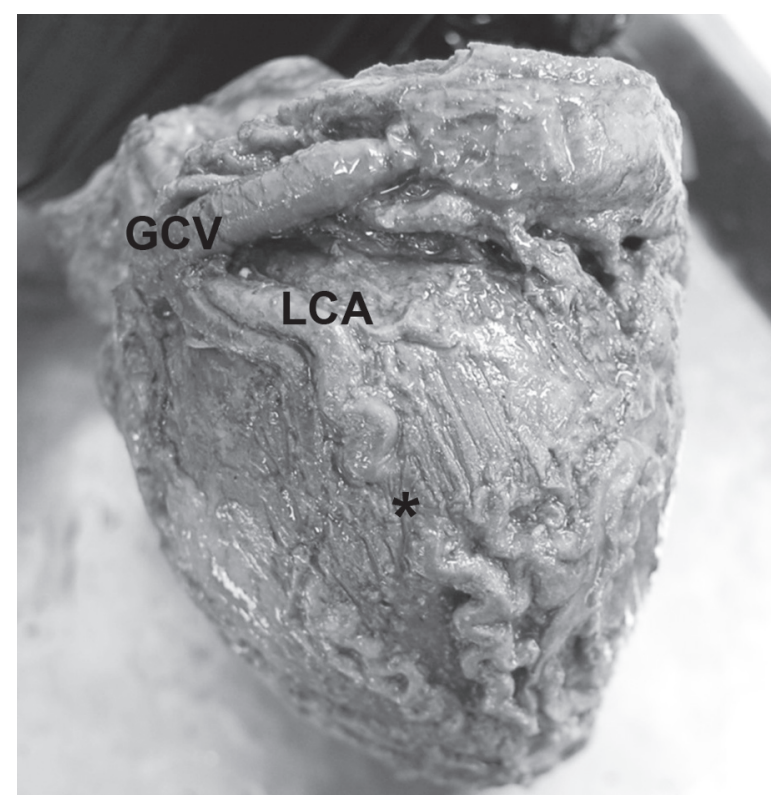

Figure 2. The heart from the left showing the near vertical orientation of the terminal branch of the left circumflex artery (LCA) and myocardial bridging $\left({ }^{*}\right)$; GCV — great cardiac vein.

of the heart the posterior interventricular artery was absent and no other arteries were found to be travelling near its usual territory (Figs. 1, 3).

\section{DISCUSSION}

The posterior interventricular artery normally supplies a small part of the posterior interventricular septum and the posterior aspect of the right ventricle. James and Burch [7] have demonstrated that the left anterior descending artery supplies a large portion of

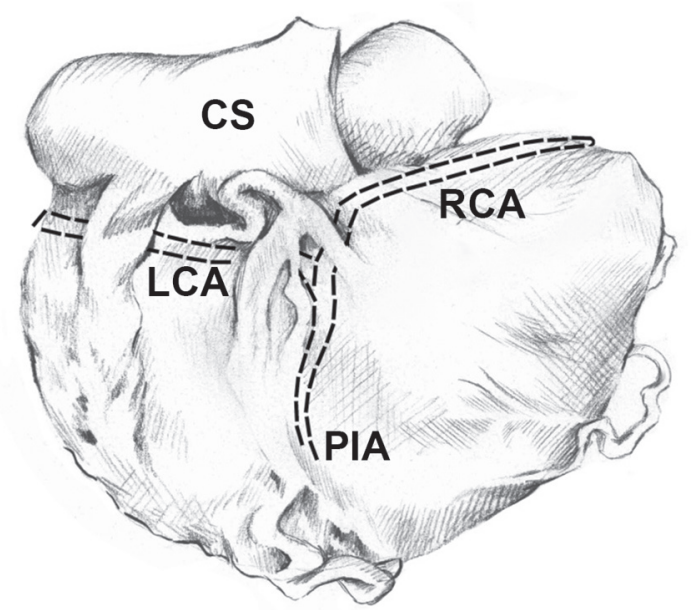

Figure 3. Schematic of the posterior aspect of the dissected specimen showing the normal expected path of either the left or right circumflex (LCA, RCA) artery to give rise to the (absent) posterior interventricular artery (PIA); CS — coronary sinus.

the interventricular septum with its deep penetrating branches. It would not be too much of a stretch to suggest that the left anterior descending artery may, in this case, have developed even deeper branches to supply the entirety of the septum. Indeed we have already seen that the left anterior descending artery in this case extends around the apex and onto the posterior aspect of the heart. With the left anterior descending artery already supplying such important parts of the heart, adding additional heart territory to its supply area may mean that an occlusion would be more likely to result in fatality.

Other cases of absent coronary arteries have been reported in the literature, including a recent case of an absent right coronary artery in a 46-year-old woman [8]. In this case, there was collateral supply by a dominant left circumflex artery that ran along the atrio-ventricular groove in which the right coronary artery usually runs. Similar cases have been described by Alhasan et al. [2] and Almansori [3]. In the cases reported by Tanawuttiwat et al. [8] and Alhasan et al. [2], both patients experienced episodes of chest pain, but also had several other cardiovascular risk factors. In the case reported by Almansori [3], the patient was a young man being investigated for a patent ductus arteriosus, with no risk factors or reason for investigation disclosed. While being normally asymptomatic, variations in coronary blood supply may predispose individuals to angina. However none of these patients was said to be completely lacking a vessel in the normal coronary distribution, which 
suggests that the variation in their coronary blood supply may not have been functionally as significant as that described here. Abnormalities such as these reinforce the importance of cardiovascular health, since most of them are initially asymptomatic and often incidental findings.

\section{ACKNOWLEDGEMENTS}

The authors would like to thank Rachel Mead for kindly providing the drawing that appears as Figure 3.

\section{REFERENCES}

1. Alegria JR, Herrmann J, Holmes DR Jr, Lerman A, Rihal CS (2005) Myocardial bridging. Eur Heart J, 26: 1159-1168.

2. Alhasan RS, Qaqi O, Elder MD (2012) An absent right coronary artery anomaly. Cath Lab Digest, 20: 46-48.
3. Almansori M (2013) Congenitally absent right coronary artery. Kardiol Pol, 71: 1295.

4. Angelini $P$ (2007) Coronary artery anomalies, an entity in search of an identity. Circulation, 115: 1296-1305.

5. Earls JP (2006) Coronary artery anomalies. Tech Vasc Interv Radiol, 9: 210-217.

6. Haddad F, Hunt SA, Rosenthal DN, Murphey DJ (2008) Anatomy, physiology, aging, and functional assessment of the right ventricle. Circulation, 117: 1436-1448.

7. James TN, Burch GE (1958) Blood supply of the human interventricular septum. Circulation, 17: 319-396.

8. Tanawuttiwat T, Harindhanavudhi T, Trivedi D (2009) Anomalous singe coronary artery with absent right coronary artery diagnosed with the aid of 64-slice multidirector computed tomographic angiography. Tex Heart Inst J, 36: 362-364.

9. Ugalde H, Ramirez A, Ugalde D, Farias E, Silva AM (2010) Coronary artery origin anomalies. Analysis of 10000 coronary angiographies. Rev Med Chil, 138: 7-14. 